\title{
Mating system drives negative associations between morphological features in Schistosomatidae
}

\author{
Sophie Beltran ${ }^{1 *}$, Yves Desdevises $^{2}$, Julien Portela ${ }^{1}$, Jérôme Boissier ${ }^{1 *}$
}

\begin{abstract}
Background: Sexual morphological features are known to be associated with the mating systems of several animal groups. However, it has been suggested that morphological features other than sexual characteristics could also be constrained by the mating system as a consequence of negative associations. Schistosomatidae are parasitic organisms that vary in mating system and can thus be used to explore links between the mating system and negative associations with morphological features.

Results: A comparative analysis of Schistosomatidae morphological features revealed an association between the mating system (monogamous versus polygynandrous) and morphological characteristics of reproduction, nutrition, and locomotion.

Conclusions: The mating system drives negative associations between somatic and sexual morphological features. In monogamous species, males display a lower investment in sexual tissues and a higher commitment of resources to tissues involved in female transport, protection, and feeding assistance. In contrast, males of polygynandrous species invest to a greater extent in sexual tissues at the cost of reduced commitment to female care.
\end{abstract}

\section{Background}

A mating system reflects the manner in which members of an animal society are structured with respect to sexual behaviour. Three mating systems are generally recognised: monogamy, polygamy, and polygynandry (or promiscuity). In monogamous species, males and females have only one sexual partner at any given time. In polygamous species, one male has a mating relationship with several females (i.e., polygyny) or one female has a mating relationship with several males (i.e., polyandry). Finally, polygynandry is a mating system in which any male mates with any female. Specific morphological features are known to be associated with the mating systems of several animal groups, including primates [1,2], bats [3], birds [4-6], rodents [7], teleost fishes [8], amphibians [9], and insects [10,11]. Logically, as a consequence of sexual selection, such

\footnotetext{
* Correspondence: beltran.sophie@hotmail.fr; boissier@univ-perp.fr 'Laboratoire de Parasitologie Fonctionnelle et Evolutive, Centre de Biologie et d'Écologie Tropicale et Méditerranéenne, UMR 5244 CNRS-UPVD, 52 Avenue Paul Alduy, 66860 Perpignan Cedex, France Full list of author information is available at the end of the article
}

morphological features mainly involve primary or secondary sexual characteristics. However, it has been suggested that morphological features other than such characteristics could also be constrained by the mating system, reflecting evolutionary trade-offs between effective mating and bodily phenotype [3]. Previous authors indicated that males of bat species with mating systems based on female promiscuity had smaller brains and larger testes, whereas species with mating systems involving female fidelity were endowed with larger brains and smaller testes. This pattern was interpreted as an investment trade-off between two metabolically expensive organs [3]. Such an "expensive sexual tissue" hypothesis proposes that more intense sexual selection will affect the evolution of energy-demanding tissue and associated functions as a result of negative association with costly sexual organs, ornaments or armaments [3]. Although this hypothesis has been proven in bats [3], no such link has been demonstrated in mammals [12].

Schistosomes (Trematoda: Schistosomatidae) are endoparasites of birds and mammals [13]. The $\sim 100$ species of schistosomes are unusual among the $\sim 18,000$ 
species of the subclass Digenea because, unlike other digeneans (which are usually hermaphroditic), schistosomes are of two separate sexes. More importantly, schistosomes are the only parasitic organisms that show variability in mating systems. Three such systems have been identified in these worms [14]: (1) Monogamy occurs in $\sim 30$ species and, in these species, worm pairs consisting of only one female and only one male can be observed either in vivo or after experimental recovery. Moreover, the monogamous female needs the continuous presence of a male to maintain sexual activity, making monogamy compulsory. However, monogamy does not imply faithfulness. Mate changes can occur, as have been shown in the genus Schistosoma $[15,16]$; this means that schistosomes are socially but not genetically monogamous [17]. (ii) Polygyny occurs in $\sim 4$ species and, in these species, one male monopolizes more than one female, with other males having no access to these females. (iii) Polygynandry occurs in $~ 66$ species; males and females are never seen in copula in vivo (i.e., males and females mate with several partners of the opposite sex over a given period of time). In contrast to monogamous female schistosomes, polygynandrous females are able to attain sexual maturity and to lay eggs even if a male is not continuously present [18]. Schistosomes are therefore the only parasitic organisms that can be used to explore possible links between a chosen mating system and a negative association with a morphological feature. The goals of the present work are (i) to determine if, as a consequence of mate competition, male polygynandrous schistosomes invest more energy (as measured by testis size) in their reproductive organs than do monogamous males; and (ii) to establish whether any negative association between investment in sexual and somatic tissues can be identified. Our prediction was that the larger the investment in sexual tissue, the smaller would be the investment in locomotor and nutritive functions, as measured by relative sucker size and oesophagus length, respectively.

\section{Methods}

\section{Data collection}

A total of 28 species were included in this study, a number that is limited by DNA sequence information required for the phylogenetic reconstruction used in the comparative analyses. Nineteen species from six genera are monogamous, and nine species from six genera are polygynandrous. DNA sequence information is available for only two polygynous species, which were therefore not included in the analysis. Data on morphological features were collected from published parasite descriptions; these measurements are summarized in Additional file 1, Table S1. The surface area of each organ was calculated from the length (l) and the width (w) of the organ using the ellipsis surface area formula $(1 \times \mathrm{w} \times \pi / 4)$. The relative organ length is the length of the organ divided by the body length, and the relative organ surface area is the surface area of the organ divided by the body surface area. We identified three groups of morphological features according to their functions (Figure 1):

1. The "reproduction group" constitutes sexual morphological features of female and male schistosomes. For females, we recorded the relative seminal receptacle surface area (seminal receptacle surface area divided by the total surface area of the body) and the relative ovary length (ovary length divided by the overall length of the body). For males, we determined the number of testes,
A.

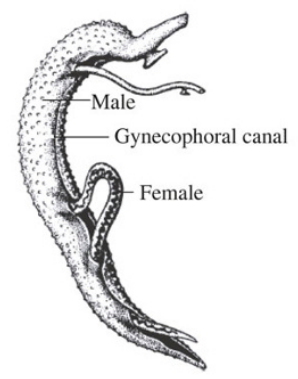

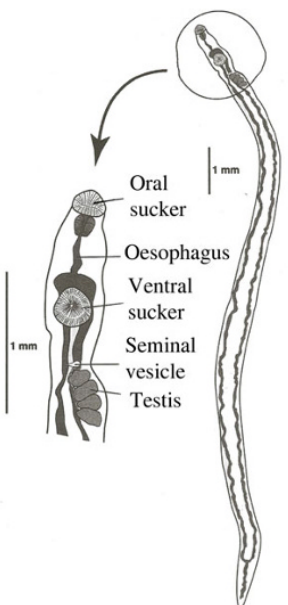

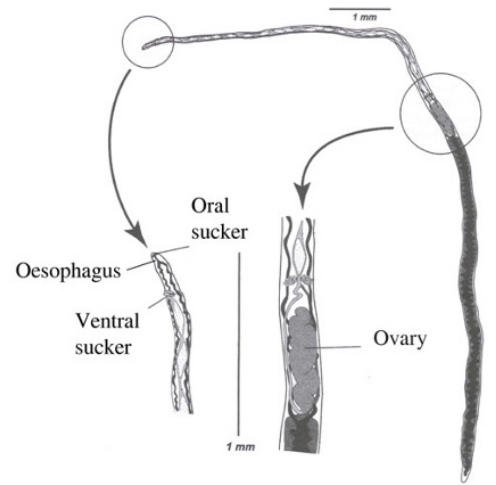

Figure 1 Morphological features recorded. A. Schistosome pair. B. Male schistosome C. Female schistosome 
and measured the relative seminal vesicle surface area (seminal vesicle surface area divided by the total surface area of the body) and relative testes surface area (total testes surface area divided by body surface area). We also recorded the relative male gynecophoral canal length (length of the gynecophoral canal divided by the overall length of the body). The gynecophoral canal is a groove on the ventral surface of the male in which the female is held during copulation.

2. The "nutrition group" constitutes somatic morphological features of female and male schistosomes involved in nutrition. For males and females, we recorded the relative oesophagus length (oesophagus length divided by the overall length of the body), which has implications for the transport of food toward gut caecae.

3. The "locomotion group" contains somatic morphological features of female and male schistosomes involved in locomotion. Schistosomes, like all digeneans, possess an oral sucker and a ventral sucker, or acetabulum. Locomotion is achieved by alternate attachment of the suckers on internal host surfaces [19]. For males and females, we measured relative oral and ventral sucker surface areas (sucker surface area divided by the total surface area of the body). We also computed male/ female relative sucker-surface-area ratios.

Note that, in addition to its inclusion in the reproduction group, the male gynecophoral canal could appear in all three morphological groups because of its potential involvement in female nutrition (through transtegumental transfer of substances) [20], female sexual maturation [21], female locomotion [22] and possibly mate guarding and female protection against the host immune system $[14,23]$.

\section{Comparative analyses}

To control for phylogeny, we performed a phylogenetic reconstruction among the Schistosomatidae species using published DNA sequences of complete $18 \mathrm{~S}$ and $28 \mathrm{~S}$ rDNA genes, and a partial sequence of the cytochrome oxidase 1 (CO1) mtDNA gene (see Additional file 2, Table S1). Sequences were aligned using MAFFT, version $5[24,25]$, and were improved by eye using $\mathrm{Se}-\mathrm{Al}$ v2.0a11 [26]. After deleting ambiguous regions from the alignments, the final lengths of DNA sequences were $1653 \mathrm{bp}$ (18S), $3741 \mathrm{bp}$ (28S) and $1095 \mathrm{bp}$ (CO1). Because not all species investigated were sequenced for all genes used, we constructed trees from the various datasets and combined these source trees via a supertree with the aid of Rainbow [27], using matrix representation with parsimony and the Baum [28] and Ragan [29] coding scheme $[30,31]$. The combined matrix was subjected to a parsimony analysis with the heuristic algorithm implemented in PAUP*, using 10 random addition replicates and the tree bisection-reconnection branch-swapping algorithm [32]. Source trees were built via Bayesian analysis with MrBayes 3.1.2 [33] by running four chains of $10^{6}$ generations. The best evolutionary models were chosen by applying a hierarchical likelihood-ratio test using MrModelTest 2.2 [34] for the rDNA sequences, and applying a mixed model to translated mtDNA sequences. The burn-in value was set to $20 \%$ of the sampled trees (1\% of the number of generations). Following Loker and Brant [13], Griphobilharzia amoena was used as the outgroup.

Comparisons of morphological features in relation to monogamous versus polygynandrous mating system were analyzed statistically using non-parametric Mann-Whitney U-tests. We also performed variation partitioning $[35,36]$ of these morphological features between historical (phylogeny) and potentially adaptive (mating system) components. The objective of this analysis is to estimate the fraction of the variation linked to the mating system (the potentially adaptive component), the fraction linked to phylogeny (the historical component), and the fraction linked to both phylogeny and the mating system (the overlap between the two components) for each morphological trait examined. This partitioning technique allows the user to compute the fraction of the variation of the response variable due to each explanatory trait under study (here, mating system and phylogenetic effects) while controlling other(s). This leads to "pure" fractions (here, fractions explained only by the mating system or only by the phylogeny), as well as a common fraction of the variation due simultaneously to both independent traits. We stress that this common fraction (here, the joint variation explained by mating system and phylogeny) is not equivalent to an interaction term in an analysis of variance. This overlap is usually considered to be phylogenetic niche conservatism (sensu Grafen [37]), reflecting the fact that the putative effect of the mating system on morphological features is intermingled with phylogenetic effects if species with the same mating system are closely related. Such variation in decomposition requires the quantification of trait variation due to phylogeny alone. This precludes the use of classical comparative methods, such as independent contrasts $[38,39]$, because such methods cannot quantify phylogenetic inertia per se (see [40]). Here, the expression of the phylogenetic variance is carried out via a principal coordinate analysis on the distance matrix computed from the phylogenetic tree of the species considered. A few principal coordinates were chosen using a broken-stick model [41] to account for phylogeny. Details of the partitioning method used, which is based on the combination of $R^{2}$ values resulting from different regressions, can be found in Desdevises et al. [35] and Cubo et al. [42]. Adjusted $R^{2}$ values, 
which have been shown to be better in a variation-partitioning context, were used here [43]. Principal coordinate analyses were performed using DistPCoA [44]. Variation partitioning and tests of significance of the fractions were computed using the functions "varpart" and "anova.cca" from the "vegan" library [45] of the $\mathrm{R}$ statistical language ( $\mathrm{R}$ Development Core Team 2008; $R:$ a language and environment for statistical computing. R Foundation for Statistical Computing, Vienna, Austria. URL http://www.R-project.org). All tests were performed using permutational procedures (9999 permutations/ test). The mating system was coded as a binary variable $(0 / 1)$. In the phylogeny obtained (see below), species were split into two clades-one containing the monogamous species, and the other containing the polygynandrous species. This design does not allow a proper test of whether a transition toward a given mating system is associated with a change in a morphological feature, because the most parsimonious explanation suggests that only one transition in mating system occurred (see [46]). We then computed the principal coordinates within each monophyletic group to test if having a certain mating system is related to modifications in given morphological features, while taking phylogeny into account.

\section{Results}

\section{Phylogeny}

The supertree analysis led to 14 equally parsimonious trees that were combined by consensus into a majority rule. The consensus was congruent with the tree obtained from phylogenetic analysis of 28s rDNA sequences. Because branch lengths were desirable for the subsequent statistical analysis, based on this phylogenetic tree, we then kept this $28 \mathrm{~S}$ rDNA tree, where we collapsed some clades as polytomies as obtained in the supertree consensus, and added the taxa from which $28 \mathrm{~S}$ rDNA sequences were missing (Schistosoma guineensis, S. edwardiense, S. hippopotami) (Figure 2). Branch lengths for these three species were estimated from the phylogenetic analysis based on CO1 mtDNA gene, and resized to be coherent with the lengths computed from the $28 \mathrm{~S}$ rDNA analysis. This tree was used for the variation partitioning analyses.

\section{Comparative analyses}

In the "reproduction group" of features (Figure 3A), males in monogamous species possessed fewer testes, showed lower relative surface areas of both testes and seminal vesicles, but had higher relative gynecophoral canal lengths than did males of polygynandrous species. Both the variation-partitioning mating system and phylogenic analyses showed that all of relative testis surface area, testis number, and relative gynecophoral canal length, were significantly linked to the mating system, with $\mathrm{R}^{2}$ values greater than 0.4 (i.e., explaining more than $40 \%$ of the variance). Females of monogamous species displayed relatively lower seminal receptacle surface areas than did polygynandrous females. However, no significant association was found between this variable and the chosen mating system. Similarly, no difference in relative ovary length among females differing in mating system was observed.

Turning to the "nutrition group" of features (Figure 3B), both males and females of polygynandrous species displayed longer relative oesophagus lengths than did monogamous species. Variation-partitioning analysis suggested that this morphological feature was significantly linked to the mating system, in both sexes.

In the "locomotion group" of features (Figure 3C), males of monogamous species displayed a higher relative sucker surface area than did males of polygynandrous species. There was no difference in sucker surface area between females of monogamous and polygynandrous species. Comparative analyses suggested a significant effect of mating system only on the male/female relative sucker surface area ratio. Thus, sexual dimorphism in sucker surface area was greater in monogamous than in polygynandrous species.

\section{Discussion}

It is now well established that, as a consequence of sperm competition, males displaying promiscuous sexual behaviour need to invest more energy in the reproductive organs than do monogamous males [47]. Such a link has been shown in primate, bird, rodent, amphibian, and insect species, and also between different populations of the same species [47]. In parasitic organisms, an impact of sexual selection on morphological features has been demonstrated in polygamous acanthocephalans [48]. In the cited study, it was shown that investment in testicular volume was related to the intensity of malemale competition. Our present work provides the first evidence from a parasitic organism showing that the development of sexual tissue is dependent on the mating system, with polygynandrous male schistosomes investing more energy in reproductive organs (measured by testis size) than do monogamous males. Literature reports on the link between accessory gland size and sperm competition level are few. Recently, it was shown in rodents that the masses of both the seminal vesicle and the anterior lobe of the prostate vary positively with testis weight [7]. Without controlling for phylogeny, we found a similar link between the relative testis and vesicle surface areas in males and the associated relative seminal receptacle surface area in females. Unfortunately, variation-partitioning tests did not show any effect of mating system on the sizes of these accessory 


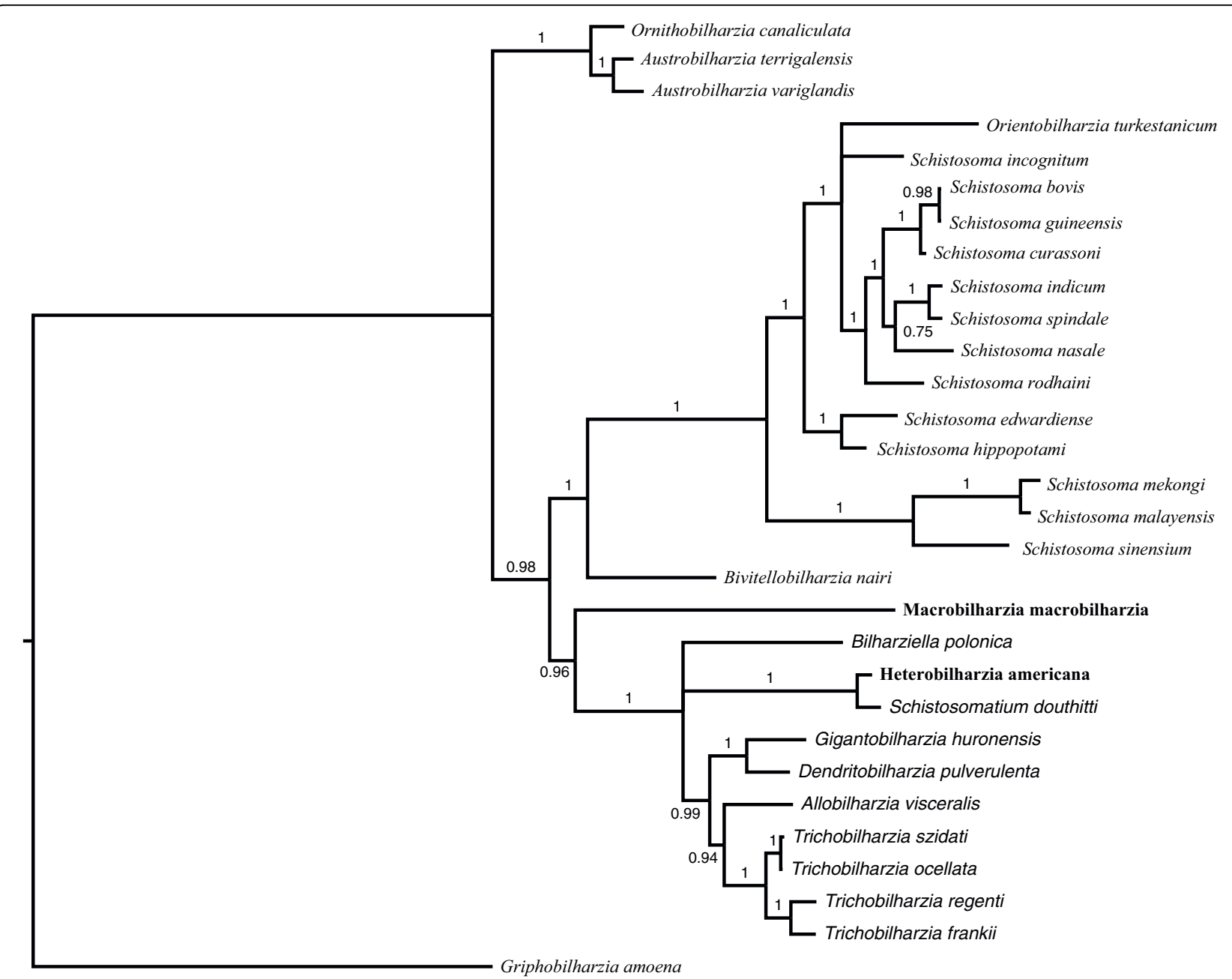

Figure 2 Phylogenetic supertree of several species from the family Schistosomatidae, obtained from phylogenetic analyses based on partial 185 and 28S rDNA, and CO1 mtDNA. Numbers near branches are posterior probabilities indicating clade support. These numbers and branch lengths were computed using Bayesian inference based on $28 \mathrm{~S}$ rDNA sequences (see text for details). Species in regular, bold and italic characters are polygynandrous, polygynous and monogamous, respectively.

sex organs, suggesting that more species need to be included in future analysis.

The gynecophoral canal, a ventral groove in which the female resides, is a male secondary sexual characteristic specific to Schistosomatidae. We found that monogamous male schistosomes had gynecophoral canals 7-fold longer than those of polygynandrous males ( $90 \%$ vs. $12 \%$ of total body length), a difference that can be fully explained by variation in mating systems. When such systems were not considered in previous studies, a negative association was observed between the size of the gynecophoral canal and the number of testes [49]. The level of paternal investment is known to be associated with the mating system [50], and it is generally accepted that the male makes a lower investment in the system when successful paternity is less likely [51]. Thus, if the gynecophoral canal represents a paternal investment, as has indeed been proposed [49], it seems logical that monogamous male schistosomes, which make a greater investment than do polygynandrous males, should possess longer canals.

In Schistosomatidae, the gonado-somatic index (i.e., the relative testis surface area) ranges from 3-24\% depending on whether the mating system is monogamous or polygynandrous. By comparison, testis mass as a percentage of body weight ranges from $0.12-8.4 \%$ in bats and from $0.02-0.75 \%$ in primates [3]. It might be expected that more energy is invested in testicular tissue, which is energetically demanding [52], less energy is available for other tissues and functions. The present study shows that if monogamous male Schistosomatidae have a lower relative testis surface area than do polygynandrous males, the relative sucker surface area is larger and the relative oesophagus length smaller. 

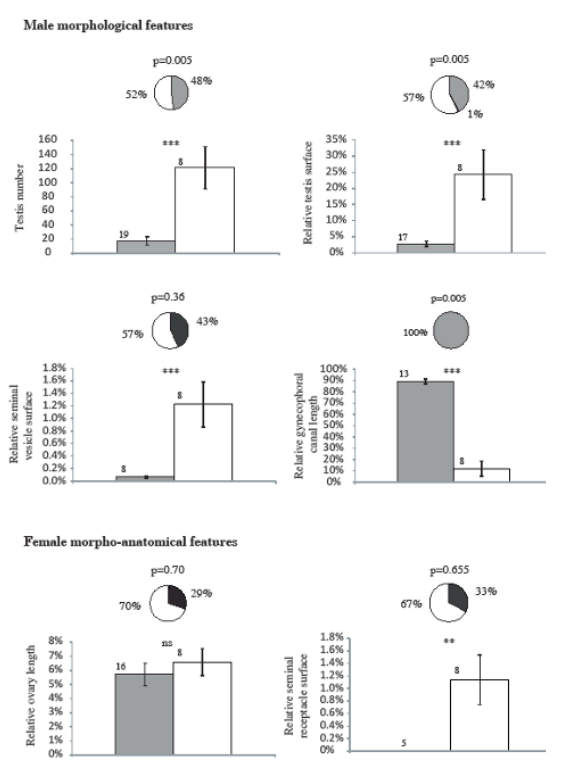

A
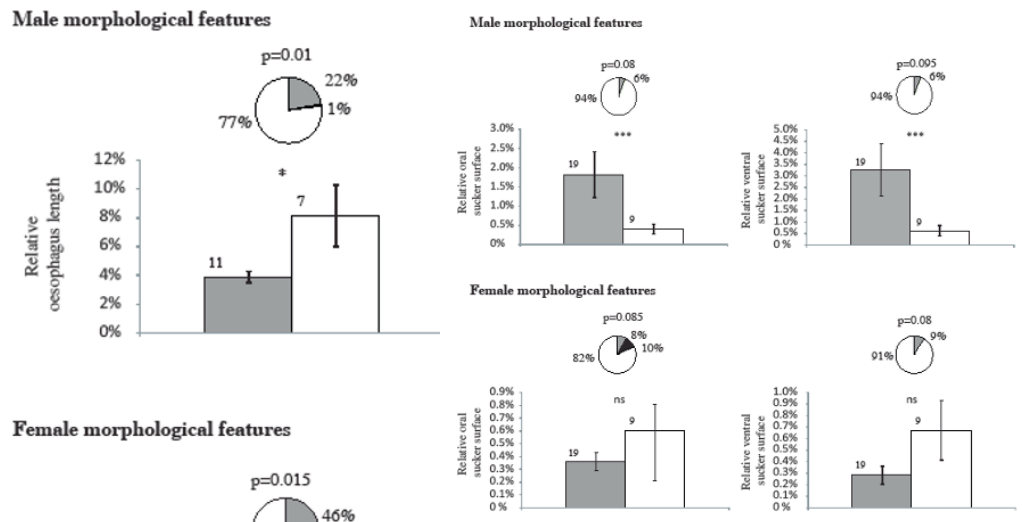

Female morphological features

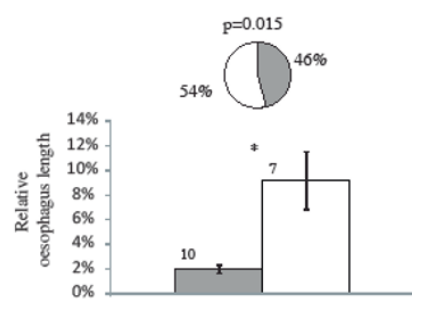

B

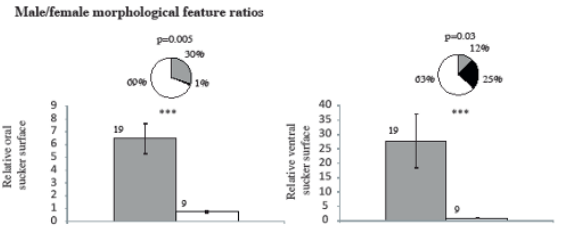

C

Figure 3 The influence of monogamous (grey histogram) versus polygynandrous (white histogram) mating systems on Schistosomatidae morphological features with implications for reproduction (A), nutrition (B) or locomotion (C). *Statistically significant difference using Mann-Whitney U-tests. Pie charts display the variation partitioning between phylogeny in white, mating system in grey, and the overlap between these two components in black. The P-value of the shares evaluation appears above the pie chart. The number above each

histogram corresponds to the sample size.

Suckers are very important organs in digeneans because the suckers allow migration and fixation of the worm in the definitive host. In addition, because Schistosomatidae are endoparasites that live in the veins of birds or mammals, the organisms must be capable of resisting blood flow. Our present work showed that relative sucker dimorphism was greater in monogamous than in polygynandrous species. This difference is a consequence of a higher relative sucker surface area in monogamous males compared to polygynandrous males, rather than a variation in relative female sucker surface area. More precisely, no difference was apparent in relative sucker surface area between monogamous females and polygynandrous male or female parasites (0.41$0.58 \%$ of body surface area when both suckers were considered). Only monogamous males displayed expanded relative sucker surface areas $(1.83 \%$ and $3.28 \%$ of body surface area for the oral and ventral suckers, respectively). This can be explained by the fact that, in monogamous species, the male parasite must maintain and transport its female to egg-laying sites. In contrast, females of polygynandrous species must travel and resist blood flow alone.

Schistosomes ingest red blood cells (the principal diet) using negative pressure created by contraction of the oral sucker muscle and the esophagus [53]. We found that the oesophagus of both male and female polygynandrous parasites was longer than that of monogamous males and females. With polygynandrous males, it may be assumed that the need to produce numerous spermatozoids requires high-level nutrient intake. In addition, because such males need not hold and transport a female, the males can invest more energy in obtaining nutrition. In polygynandrous females, the longer length of the oesophagus compared to that of monogamous females may be a consequence of the absence of continuous pairing. In monogamous schistosomes (at least in the Schistosoma genus, for which most information is available), it is well established that the male assists the female to pump blood and to reach sexual maturity [54]. A lone female is stunted and unable to produce eggs [18]. Therefore, as a consequence of the mating system, monogamous females, aided by their males, would be expected to possess a shorter oesophagus than that of polygynandrous females, which live separately from males.

\section{Conclusions}

The present study shows that the mating system drives negative associations between somatic and sexual morphological features. Monogamous males invest less in sexual tissues (the testes and associated organs) and more in tissues required for female transport, protection, 
and feeding assistance. On the other hand, polygynandrous males make a greater investment in sexual tissues and a lower investment in female care compared to monogamous males. Therefore, sexual selection acts not only on primary and secondary sex organs, but also on somatic organs, the functions of which are beneficial in a given mating system.

\section{Additional material}

Additional file 1: Table S1: Morphological features noted for each Schistosomatidae species. M, monogamous; P, polygynandrous; NA, no available data.The surface area of each organ was calculated based on the length (I) and the width ( $W$ ) of the organ using the ellipsis surface area formula $(\mid \times w \pi / 4)$. The relative organ length is the length of the organ divided by the body length, and the relative organ surface area is the surface area of the organ divided by the body surface area.

Additional file 2: Table S1: Accession numbers of the sequences used for phylogenetic reconstruction.

\begin{abstract}
Acknowledgements
We thank Serge Morand for valuable and constructive comments on the manuscript and Joan Straub for editing the English of the manuscript. This work was supported by the French Ministère de l'Enseignement Supérieur et de la Recherche, the CNRS, and the Agence Nationale de la Recherche (Program Monogamix ANR-08-BLAN-0214-02)
\end{abstract}

\section{Author details}

${ }^{1}$ Laboratoire de Parasitologie Fonctionnelle et Evolutive, Centre de Biologie et d'Écologie Tropicale et Méditerranéenne, UMR 5244 CNRS-UPVD, 52 Avenue Paul Alduy, 66860 Perpignan Cedex, France. ${ }^{2}$ FRE 3355 CNRS-UPMC, Biologie Intégrative des Organismes Marins, Université Paris 06, Observatoire Océanologique, 66650 Banyuls-sur-Mer, France.

\section{Authors' contributions}

$J B$ and JP compiled the database. YD performed the phylogenetic and the comparative analyses. SB, YD and JB drafted the manuscript. All authors read and approved the final manuscript.

Received: 15 January 2010 Accepted: 10 August 2010

Published: 10 August 2010

\section{References}

1. Harcourt AH, Harvey PH, Larson SG, Short RV: Testis weight, body weight and breeding system in primates. Nature 1981, 293(5827):55-57.

2. Schillaci MA: Sexual selection and the evolution of brain size in primates. PLOS ONE 2006, 1:e62.

3. Pitnick S, Jones KE, Wilkinson GS: Mating system and brain size in bats Proc R Soc B 2006, 273(1587):719-724.

4. Dunn PO: Forced copulation results in few extrapair fertilizations in Ross's and lesser snow geese. Anim Behav 1999, 57(5):1071-1081.

5. Moller AP, Briskie JV: Extra-paternity, sperm competition and the evolution of testis size in birds. Beh Ecol Sociobiol 1995, 36:357-365.

6. Pitcher TE, Dunn PO, Whittingham LA: Sperm competition and the evolution of testes size in birds. J Evol Biol 2005, 18(3):557-567.

7. Ramm SA, Parker GA, Stockley P: Sperm competition and the evolution of male reproductive anatomy in rodents. Proc $R$ Soc $B 2005$, 272(1566):949-955

8. Stockley P, Gage MJ, Parker GA, Moller AP: Sperm competition in fishes: the evolution of testis size and ejaculate characteristics. Am Nat 1997, 149(5):933-954.

9. Jennions MD, Passmore NI: Sperm competition in frogs: testis size and a 'sterile male' experiment on Chiromantis xerampelina (Rhacophoridae). Biol J linn Soc 1993, 50:211-220.
10. Gage MJG: Association between body size, mating pattern, testis size and sperm lenght accross butterflies. Proc $R$ Soc $B$ 1994, 258:247-254.

11. Simmons LW, Emlen DJ: Evolutionary trade-off between weapons and testes. Proc Natl Acad Sci USA 2006, 103(44):16346-16351.

12. Lemaître J, Ramm S, Barton R, Stockley P: Sperm competition and brain size evolution in mammals. J Evol Biol 2009, 22:2215-2221.

13. Loker ES, Brant SV: Diversification, dioecy and dimorphism in schistosomes. Trends in Parasitology 2006, 22(11):521-528.

14. Beltran S, Boissier J: Schistosome monogamy: who, how, and why? Trends Parasitol 2008, 24:386-391.

15. Pica-Mattoccia L, Moroni R, Tchuem Tchuente LA, Southgate VR, Cioli D: Changes of mate occur in Schistosoma mansoni. Parasitology 2000, 120:495-500

16. Beltran S, Cézilly F, Boissier J: Genetic dissimilarity between mates, but not male heterozygosity, influences divorce in schistosomes. PLOS ONE 2008, 3(10):e3328.

17. Beltran S, Boissier J: Are schistosomes socially and genetically monogamous? P Res 2009, 104:481-483.

18. Armstrong JC: Mating behavior and development of schistosomes in the mouse. J Parasitol 1965, 51:605-616.

19. Basch PF: Development and behavior of cultured Schistosoma mansoni fed on human erythrocyte ghosts. Am J Trop Med Hyg 1984, 33(5):911-917.

20. Silveira AM, Friche AA, Rumjanek FD: Transfer of [14C] cholesterol and its metabolites between adult male and female worms of Schistosoma mansoni. Comp Biochem Physiol 1986, 85(4):851-857.

21. Popiel I: Male-stimulated female maturation in Schistosoma: a review. J Chem Ecol 1986, 12:1745-1753.

22. Basch PF: Why do schistosomes have separate sexes? Parasitol Today 1990, 6:160-163.

23. Boissier J, Mone H: Male-female larval interactions in Schistosoma mansoni infected Biomphalaria glabrata. Int J Parasit 2001, 31:352-358.

24. Katoh K, Misawa K, Kuma K, Miyata T: MAFFT: a novel method for rapid multiple sequence alignment based on fast Fourier transform. Nucleic Acid Res 2002, 30:3059-3066.

25. Katoh K, Kuma K, Toh H, Miyata T: MAFFT version 5: improvement in accuracy of multiple sequence alignment. Nucleic Acid Res 2005 33:511-518.

26. Rambaut A: Se-Al: Sequence Alignment Editor. 1996 [http://tree.bio.ed.ac uk/software/seal/]

27. Chen D, Eulesnstein O, Fernandez-Baca D: Rainbow: a toolbox for phylogenetic supertree construction and analysis. Bioinformatics 2004, 16:2872-2873.

28. Baum B: Combining trees as a way of combining data sets for phylogenetic inference, and the desirability of combining gene trees. Taxon 1992, 41:3-10.

29. Ragan M: Phylogenetic inference based on matrix representation of trees. Mol Phyl Evol 1992, 1:53-58.

30. Wiens J, Reeder T: Combining data sets with different numbers of taxa for phylogenetic analysis. Syst Biol 1995, 44:548-549.

31. Sanderson M, Purvis A, Henze C: Phylogenetic supertrees: assembling the trees of life. TREE 1998, 13:105-109.

32. Swofford D: PAUP*. Phylogenetic Analysis Using Parsimony (*and Other Methods). Version 4d10. Sinauer Associates, Sunderland, Massachusetts. 2003

33. Ronquist F, Huelsenbeck J: MRBAYES 3: Bayesian phylogenetic inference under mixed models. Bioinformatics 2003, 19:1572-1574.

34. Nylander JAA: MrModeltest 2.2. Program distributed by the author. Evolutionary Biology Centre, Uppsala University 2004

35. Desdevises Y, Legendre P, Azouzi L, Morand S: Quantifying phylogenetically structured environmental variation. Evolution 2003 57(11):2647-2652.

36. Borcard D, Legendre $P$, Drapeau P: Partialling out the spatial component of ecological variation. Ecology 1992, 73:1045-1055.

37. Grafen A: The phylogenetic regression. Phil Trans $R$ Soc B 1989 326:119-157.

38. Felsenstein J: Phylogenies and the comparative method. Am Nat 1985 125:1-15.

39. Felsenstein J: Comparative methods with sampling error and withinspecies variation: contrasts revisited and revised. Am Nat 2008, 171:713-725 
40. Westoby $M$, Leishman $M$, Lord J: On misinterpreting the 'phylogenetic correction'. J Ecol 1995, 83(3):531-534

41. Diniz-Filho J, de Sant' Ana C, Bini L: An eigenvector method for estimating phylogenetic inertia. Evolution 1998, 52:1247-1262.

42. Cubo J, Legendre P, de Ricqles A, Montes L, de Margerie E, Castanet J, Y D: Phylogenetic, functional, and structural components of variation in bone growth rate of amniotes. Evol Dev 2008, 10(2):217-227.

43. Peres-Neto $P$, Legendre P, Dray S, Borcard D: Variation partitioning of species data matrices: estimation and comparison of fractions. Ecology 2006, 87:2614-2625.

44. Legendre P, Anderson M: Program DistPCoA. Département de sciences biologiques, Université de Montréal. 1998.

45. Oksanen J, Kindt R, Legendre P, O'Hara R: vegan: Community Ecology Package version 1 8-5. 2007 [http://cran.r-project.org/].

46. Garland T Jr, Bennett AF, Rezende EL: Phylogenetic approaches in comparative physiology. J Exp Biol 2005, 208(Pt 16):3015-3035.

47. Parker GA: Sperm competition and the evolution of ejaculates: towards a theory base. Sperm competition and sexual selection, Academic Press; LondonBirkhead TR, Møller AP 1998, 3-54.

48. Sasal $P$, Jobet $E$, Faliex $E$, Morand S: Sexual competition in an acanthocephalan parasite of fish. Parasitology 2000, 120(Pt 1):65-69.

49. Morand S, Muller-Graf CD: Muscles or testes? Comparative evidence for sexual competition among dioecious blood parasites (Schistosomatidae) of vertebrates. Parasitology 2000, 120(Pt 1):45-56.

50. Moller PA, Cuervo JJ: The evolution of paternity and paternal care in birds. Behav Ecol 2000, 11:472-485.

51. Neff BD: Decisions about parental care in response to perceived paternity. Nature 2003, 422(6933):716-719.

52. Kenagy GJ, Trombulak SC: Size and function of mammalian testes in relation to body size. J Mammal 1986, 67:1-22.

53. Maule AG, Marks NJ: in Parasitic Flatworms: Molecular Biology, Biochemistry, Immunology and Physiology. Queen's University, Belfast, Northern Ireland 2006.

54. Basch PF: Schistosomes. Development, reproduction, and host relations. New-York: Oxford University Press 1991.

doi:10.1186/1471-2148-10-245

Cite this article as: Beltran et al:: Mating system drives negative associations between morphological features in Schistosomatidae. BMC Evolutionary Biology 2010 10:245.

\section{Submit your next manuscript to BioMed Central and take full advantage of:}

- Convenient online submission

- Thorough peer review

- No space constraints or color figure charges

- Immediate publication on acceptance

- Inclusion in PubMed, CAS, Scopus and Google Scholar

- Research which is freely available for redistribution

Submit your manuscript at www.biomedcentral.com/submit
Biomed Central 